Review Article

\title{
Importance of Mechanical Periodontal Therapy in Patients with Diabetes Type 2 and Periodontitis
}

\author{
Priscilla Naiff $\mathbb{D}^{\mathbb{D}},{ }^{1}$ Valéria Carneiro, ${ }^{2}$ and Maria do Carmo Guimarães ${ }^{2}$ \\ ${ }^{1}$ Ph.D. Student, Faculty of Health Sciences, University of Brasilia, Distrito Federal, Brazil \\ ${ }^{2}$ Ph.D. Professor at Periodontics Division, University of Brasilia, Distrito Federal, Brazil \\ Correspondence should be addressed to Priscilla Naiff; pri_naiff@yahoo.com
}

Received 27 July 2018; Accepted 29 August 2018; Published 25 September 2018

Academic Editor: Maha El Tantawi

Copyright (c) 2018 Priscilla Naiff et al. This is an open access article distributed under the Creative Commons Attribution License, which permits unrestricted use, distribution, and reproduction in any medium, provided the original work is properly cited.

Periodontitis is an infectious and inflammatory disease of high prevalence worldwide and constitutes a significant oral health problem. It can lead to tooth loss. In addition, the local inflammatory process can cause the release of inflammatory mediators in the bloodstream and, consequently, contribute to the emergence of systemic effects as cardiovascular and diabetic complications. The purpose of this mini review is to alert health professionals about the risk that periodontitis represents for the onset or exacerbation of complications in individuals with type 2 diabetes mellitus and to emphasize that the mechanical treatment of periodontal disease and reestablishment of oral health are essential for the metabolic control of these patients. The periodontal therapy may help to reduce the risk of systemic complications in diabetes patients. Proper dental management should be suggested by health professionals, mainly from physicians to their patients, in order to improve the health conditions in these individuals.

\section{Introduction}

Diabetes mellitus (DM) is a metabolic disease in which the body does not produce insulin or cannot use it properly. There is an estimative that there are about 422 million people with DM worldwide [1].

The two main forms of the disease are type 1 (DM1) and type 2 (DM2) diabetes. Besides, other forms are also described in the literature, such as gestational diabetes, as well as other specific types of DM such as those associated with genetic disorders, among other factors. However, DM1 and DM2 affect most of the population, where $90 \%$ of the disease's cases are concentrated in type 2 [2].

In type $1 \mathrm{DM}$, beta-pancreatic cells are mistakenly attacked by the human's immune system. So, insufficient or no insulin is released. Consequently, glucose stays in the blood instead of being used as energy by the body [2].

Because most of the studies about the relation of DM and periodontitis are related to DM2 and this is the most prevalent type of diabetes, this review will approach the aspects only involved in the treatment of periodontitis in Diabetes mellitus type 2 patients.
DM2, a global public health problem, consists of a heterogeneous group of metabolic disorders that presents chronic hyperglycemia as a result of defects in the action or the insulin secretion. DM2 results from a combination of insulin resistance and inadequate compensatory response to insulin secretion, leading to a relative deficiency in the release of this hormone [3]. Insulin is the only hormone responsible for the reduction of blood glucose and is produced and released by the $\beta$-cells of the pancreatic islets of Langerhans [4].

The major complications of diabetes are microangiopathy, nephropathy, neuropathy, macrovascular disease, and delayed wound healing. Periodontitis is considered the sixth complication of DM [2].

Periodontitis is a primarily infectious and inflammatory disease caused by anaerobic bacteria (Porphyromonas gingivalis, Treponema denticola, Prevotella intermedia, Prevotella nigrescens, Eikenella corrodens, Aggregatibacter actinomycetemcomitans, among others) in association or not with other periodontopathogens, in dental biofilm. It affects teeth's protection and support tissues as gingiva and alveolar bone and can lead to dental mutilation $[1,5]$.

Periodontitis has been recently very strongly associated with the role of an overreactive immune system in the critical 
point of the periodontal disease development [6, 7]. Periodontopathogens stimulate, among other inflammatory mediators, the production of cytokines as interleukin 1 beta (IL1$\beta$ ), interferon gamma (IFN- $\gamma$ ), tumor necrosis factor-alpha (TNF- $\alpha$ ), and chemokines (CXCL-1, CXCL-8, CCL-5) by gingival epithelium, adhesion molecules, increased permeability of the gingival capillaries, and chemotaxis of neutrophils from the junctional epithelium to the gingival sulcus. This initial response, with the production of specific cytokines and chemokines, promotes the migration of an inflammatory infiltrate composed of perivascular T cells and monocytes to the connective tissue $[6,7]$. If the cellular immune response fails to control the infection, recruitment of $\mathrm{B}$ cells that differentiate into plasma cells occurs. Plasma cells produce immunoglobulins (antibodies) that may confer protection to periodontal tissues, control the infectious process, or induce deleterious effects, leading to the destruction of connective tissue and promoting reabsorption of the alveolar bone. The effectiveness of this response varies between individuals and demonstrates importance in determining disease susceptibility [6].

Nowadays is well established the relationship between the progression of periodontitis and several factors, such as the presence of the periodontal pathogens, high levels of proinflammatory cytokines (IFN- $\gamma$ and TNF- $\alpha$ ), matrix metalloproteinases (MMP) and prostaglandin $\mathrm{E}_{2}\left(\mathrm{PGE}_{2}\right)$, and low levels of inflammation's inhibitory cytokines (IL$10)$, transforming growth factor beta (TGF- $\beta$ ), and tissue inhibitors of MMP (TIMP) [6, 8].

The susceptibility and extent of tissue destruction appear to be determined by the complex cytokine balance produced by the presence of numerous associations between periodontal microorganisms. When the host's response is exacerbated, it can lead to tissue damage, causing loss of periodontal support [6, 7].

Systemic diseases, such as diabetes, can also interfere with the periodontal condition, turning the prognosis of the associated diseases unfavorable [9]. Intravascular dissemination of microorganisms and their products throughout the body may occur because of the inflammation in the periodontal tissues. The total surface area of this periodontal inflammatory field is estimated to be the size of the palm of the hand. Immediate medical intervention should be done if one lesion of this size was on the skin. However, periodontitis is frequently ignored by health professionals, even though it may be associated with a range of systemic diseases and conditions [10].

The association of periodontitis with DM has been investigated and studies have shown that there is a definite correlation between both [11]. It can be observed that individuals with diabetes and inadequate glycemic control are more likely to develop severe periodontal disease and that periodontitis may interfere in the glycemic control of these individuals $[12,13]$.

\section{Mechanisms of Action}

Patients with uncontrolled diabetes can present with microor macrovascular complications. The prevalence of both complications occurs according to the type and duration of DM. Microvascular defects affect most intricately vascularized organs, as the retina (retinopathy), kidney (nephropathy), and peripheral nerves (neuropathy). The macrovascular defects affect large blood vessels, and consequently, some noble organs as the heart (cardiovascular disease), brain (cerebrovascular disease), and the peripheral arteries (peripheral vascular disease) [14]. Vascular disorders are usually progressive. The main problem in uncontrolled diabetes is the activation of the immune system that leads to micro- and macroangiopathies and other immune reactions contributing to all major organs failure. For example, nephropathy, begins insidiously, but over time, may contribute significantly to morbidity and mortality, resulting in severe damage to the organs. Cardiovascular diseases (CVDs) also account for increased morbidity and mortality in DM2 patients [15].

CVD usually occurs about two decades earlier among DM2 patients than in those without the disease [16, 17]. About $70 \%$ of individuals with DM2 die of CVD [18]. The combination of the duration of diabetes ( $>15$ years) and prior CVD was associated with a 30-fold increased risk of fatal CVD [19].

Several factors can explain the mechanisms of periodontal destruction due to DM. Initially, the hyperglycemia state can directly favor the growth of periodontal pathogens, hinder or prejudice cellular functions and, consequently, host defenses. One pathogenic consequence of hyperglycemia in diabetes is an insufficiency in detoxification of reactive carbonyl compounds. Reactive carbonyl increases due to oxidative and nonoxidative reactions where carbohydrates and lipids lead proteins to chemical modification and then, at a late stage, to oxidative stress and tissue damage. This chronic and accelerated chemical modification of proteins is associated with the AGEs (advanced glycation end products) hypothesis which proposes that by increasing concentration of glucose in diabetes alters the structure and function of tissue proteins, contributing and precipitating the development of diabetic complications [20].

The immunological mechanisms mediating the effect of periodontitis on the control of diabetes have moderated level of support in the current literature. Most studies demonstrate that circulating proinflammatory mediators as TNF- $\alpha$, CRP, and mediators of oxidative stress are elevated in patients with both diseases and these subjects tend to demonstrate higher dyslipidemia, reduced beta cell function, and elevated oxidative stress (that may act synergistically in worsening cardiovascular complications in diabetes) than patients with diabetes alone. Probably these mediators affect the control of DM, but there is no sufficient information from animal studies to support this possibility [21].

There is also a substantial increase in mediators as proinflammatory cytokines and secretion of collagendegrading enzymes. Diabetes, through the formation of AGEs, can indirectly alter the union of the extracellular matrix, as well as cellular activities, amplifying inflammatory reactions, and decreasing cellular viability, which leads to deterioration of the healing process and potential change in periodontal tissues [22]. 
On the other hand, the mechanisms by which periodontitis promotes metabolic dysfunction are not yet fully understood. It is believed that in response to endotoxins such as lipopolysaccharide (LPS) produced by periodontal microorganisms, there is an augment in the production of proinflammatory cytokines, chemokines, reactive oxygen species (ROS), and C-reactive protein (CRP) that can alter lipid metabolism and insulin resistance, leading to hyperlipidemia and hyperglycemia [22]. Additionally, TNF- $\alpha$ has been identified as a potent insulin receptor blocker [23].

In severe untreated periodontitis, the ulcerated epithelium of the periodontal pockets has an estimated surface area of 8 to $20 \mathrm{~cm}^{2}$ [24]. This inflamed and ulcerated subgingival epithelial area of periodontal pockets constitutes a vast portal of entry for periodontopathogenic bacteria, their products, endotoxins such as LPS, and stimulated inflammatory mediators to reach the systemic circulation $[25,26]$.

Periodontal microorganisms as well as their antigens, when systemically dispersed, can cause significant systemic inflammation and contribute to DM complications. Leukocytes, endothelial cells, and hepatocytes respond to virulence factors with the secretion of proinflammatory mediators such as cytokines, chemokines, ROS, and CRP. If excessive, ROS release by phagocytes can reach circulation and cause systemic oxidative stress. CRP is a protein mainly produced by the liver as result of increased levels of TNF- $\alpha$ and IL-6 in the inflammatory process [27]. Cardiovascular disease has CRP as an independent predictor of its occurrence [28].

Data from a systematic review [29] concluded that human studies, animal experiments, and ex vivo cell culture studies provide evidence for elevated levels of interleukin- 6 and interleukin- $1 \beta$ in periodontal tissues and crevicular fluid in patients with DM and periodontitis compared to systemically healthy patients.

Animal models with type 2 diabetes mellitus suggest that TNF- $\alpha$ plays an essential role in prolonging periodontal inflammation [29] and in the development of insulin resistance [23]. This mediator reduces the expression of glucose transporter type 4 (GLUT4) which is an insulinregulated glucose transporter. TNF- $\alpha$ also induces serine phosphorylation of insulin receptor substrate-1 (IRS-1) that acts as an inhibitor of insulin receptor and down streams the signaling of phosphatidylinositol-3 kinase activation [23].

The increased release of proinflammatory cytokines (IL$1 \beta$, IL- 6 , and TNF- $\alpha$ ), an altered RANKL/osteoprotegerin ratio, interactions between advanced glycation end products and their receptors, increased production of reactive oxygen species, and increased interaction between endothelial cells and leukocytes play a crucial role in the two-way relationship between diabetes and periodontitis. These complex changes, resulting from the presence of diabetes, modify the local inflammatory reaction in the periodontium, leading to a proinflammatory state in the gingival tissue and microcirculation [29]. With continued exposure, soluble antigens react with specific circulating antibodies to form immune complexes that amplify inflammation at the sites of deposition. Likewise, proinflammatory mediators, produced locally in the inflamed gingival tissues, can reach the systemic circulation. Proinflammatory cytokines in the circulation induce leukocytosis and acute phase proteins (e.g., CRP). In this context, the increase in the number of white blood cells is associated with an augmented risk of coronary heart disease, cardiovascular disease (CVD), atherosclerosis, thrombosis, and myocardial ischemia. This increase may be caused by the inflammatory nature of chronic infections such as periodontitis [30,31].

Periodontitis may cause bacteremia and enhance atherosclerotic plaque formation because some microorganisms related to periodontal diseases were detected in atherosclerotic plaques [32, 33, 34]. However, other oral pathogens as Streptococcus mutans also have been found in atheromatous plaque samples [35]. Thus, it seems that the disruption of epithelial integrity from periodontal pockets may also provide a point of entry for nonperiodontal pathogens, as those usually found in caries-affected teeth.

Periodontal bacteria, as $P$. gingivalis, or their products can also interact with platelets (direct or via the vascular endothelium) and promote prothrombotic effects [36].

Proinflammatory cytokines, which have been reported to be associated with periodontitis, are also involved in atherothrombogenesis $[37,38]$. Furthermore, periodontitis patients present many similar risk factors to those with CVD including age, lower socioeconomic status, and smoking [39]. This suggests that periodontitis and CVD may share common etiological pathways and that the association between both is plausible.

Periodontitis is a risk factor for atherosclerosis through endothelial activation. Bacterial products (LPS, outer membrane vesicles, or fimbriae), cytokines, and chemokines resulted from the infectious and inflammatory periodontal process fall into the bloodstream and may stimulate a superregulation of endothelial cell surface receptors in addition to the expression of adhesion on vascular endothelium. This promotes chemotaxis for circulating monocytes. These cells adhere to the activated endothelium. Due to molecular mimicry, immunoglobulins against specific bacterial proteins act as autoantibodies and induce apoptosis in the endothelium. The monocytes then migrate into the subendothelial space and differentiate into macrophages. There, they pick up oxidized low-density lipoprotein (LDL) and become foam cells. Apoptosis of LDL-loaded macrophages results in the accumulation of lipids in the subendothelial space, contributing to the formation of atheromatous plaques. In addition, invading periodontal pathogens induce the proliferation of smooth muscle cells in the formation of the intima and neointima. The extracellular matrix development and the extravasation of $\mathrm{T}$ lymphocytes result in the formation of a fibrous envelope covering the atheroma. The fibrous cap and its prothrombotic components are exposed after endothelial cell apoptosis. The enzymatic degradation of the extracellular matrix results in plaque rupture with consequent exposure of its prothrombotic components and formation of thrombi, leading to vessel occlusion. Clinically, this manifest as acute myocardial infarction, in the case of an occluded coronary artery, or a stroke in the case of an occluded cerebral vessel [40]. 
On the other hand, complications of DM2 because of periodontitis can be prevented or diminished if periodontal disease is treated. Studies have demonstrated that mechanical periodontal therapy can promote the reduction of inflammation's markers in the bloodstream (CRP, IL-6, among others) [41, 42, 43].

\section{Importance of the Diagnosis of Periodontitis and Periodontal Debridement}

Periodontal diseases have been associated with a reduced glycemic control in diabetes. Periodontitis increases the risk for the diabetes incidence in nondiabetic patients [21] as well as increases insulin resistance in patients with DM and disease complications, including mortality [44, 45].

The implications of periodontitis in the oral environment and maintenance of affected teeth, by themselves, would justify the relevance of seeking the complete understanding of its etiopathogenesis and, from this, implement active forms of individualized therapy. However, in addition to the implications of the disease in oral health, its meaning reaches systemic proportions, whose mechanisms are still not precise.

The effect of periodontitis on the control of DM type 2 has been studied, and there is indirect evidence to support biological mechanisms mediating this effect as reduced pancreatic islets $\beta$-cell function, elevated oxidative stress, and dyslipidemia. People with DM and periodontitis usually have elevated circulating proinflammatory mediators, like TNF- $\alpha$, IL-6, CRP, and reactive oxygen species (ROS) that can interfere with diabetes metabolic control [44] and may act synergistically in worsening cardiovascular complications in diabetes [45].

Mechanical periodontal therapy involves the removal of bacterial agents from periodontium, supra, and subgingival calculus, by scaling and root planning with periodontal curettes or ultrasonic devices. It is the conventional treatment for periodontitis for resolution of inflammation from periodontal tissues and consequently, control of periodontal disease [46].

There is considerable evidence that nonsurgical periodontal treatment reduces oxidative stress, C-reactive protein level, and proinflammatory cytokines (i.e., tumor necrosis factor-alpha, interleukin-1 $\beta$, and interleukin-6) [21, 42, 47, 48].

To monitor the success of the treatment of periodontal disease and the resolution of inflammation before and after therapy, besides radiographic examination, the oral clinical examination must include some essential periodontal indexes which are analyzed with the aid of a periodontal probe. The parameters usually analyzed are probing depth (PD), clinical attachment level (CAL), visible plaque index (PI), and gingival bleeding on probing (BOP) index [49].

In fact, if periodontitis truly has measurable effects on general health, treatment of this infection may alter the severity of the outcomes, with the resolution of inflammation.

The importance of periodontal treatment is not only to promote the reduction of local clinical inflammation, but it has also been associated with a subsequent decrease in serum levels of IL-6, TNF- $\alpha$, CRP, and ROS [50-55]. This evidence supports the mechanistic link between periodontitis and diabetes through inflammatory mediators.

It is important to emphasize that diabetes can interfere with the homeostatic interaction between microorganisms and host at periodontal sites, where host immune response to diabetes can trigger a destructive inflammatory pathway against previously well-tolerated microorganisms. Experimental models demonstrate that the development of periodontitis in diabetic rats involves a high expression of proinflammatory cytokines (TNF- $\alpha$, IL- $1 \beta$, IL-6) and destructive tissue factors as advanced glycation end products (AGEs) without significant changes in commensal oral microbiota [56].

Patients with DM2 usually have glycated hemoglobin HbA1C elevation in serum, so current studies have shown that periodontal therapy can improve the control of $\mathrm{HbA} 1 \mathrm{C}$ levels in patients with both diseases. Periodontal treatment can also successfully reduce circulating levels of TNF- $\alpha$, CRP in patients with DM associated with periodontitis $[57,58]$; however, research about the impact of successful long-term periodontal treatment does not exist and should be done. The magnitude of reported $\mathrm{HbA} 1 \mathrm{C}$ reductions ranges from $0.27 \%$ to $0.48 \%$ at $3-4$ months following periodontal therapy [58], which means the same quantity of short-term HbA1C reduction obtained to that often achieved by adding a second medication to a pharmacological regimen [59]. If such decreases can be sustained over the longer term after periodontal therapy, it may contribute to reduced morbidity and mortality associated with DM.

It is challenging to estimate the social cost of the morbidities related to patients living with diabetes. Many individuals are unable to continue their work activities because of chronic complications of the disease or remain with some limitations in their professional performance, causing significant losses regarding productivity. Thus, the control of periodontal disease in patients with DM through mechanical debridement (scaling and root planning) is crucial and may lead to better metabolic control and, consequently, to the improvement of the quality of life of these people.

It is of extreme relevance that health-care professionals, as physicians, to be aware of periodontitis and its implications for glycemic control and complications in individuals with diabetes. Diagnosis of periodontitis should be an integral part of a diabetes care visit. A periodontal examination should be done as part of their ongoing management of DM by a dentist. Even without diagnosed periodontitis initially, an annual periodontal review is recommended. All patients with DM should be provided with oral health education as part of their overall educational program [21].

In the other hand, dentists should pay attention in identifying both prediabetes and undiagnosed diabetes mellitus because periodontitis could increase the risk of many diabetes complications as retinopathy, nephropathy, neuropathic foot ulceration, cardiovascular diseases and mortality [60].

In conclusion, periodontitis and diabetes establish a twoway pathway, and each one, if untreated, could promote or 
exacerbate complications of each other. Periodontal screening must be part of the overall clinical examination of patients with diabetes and, if diagnosed, periodontal disease must be treated appropriately to avoid or exacerbate diabetes complications besides improving glycemic control in these individuals.

\section{Additional Points}

Clinical Significance. To alert health professionals about the relation of diabetes mellitus and periodontitis and encourage them to conduct a multidisciplinary treatment/ assistance.

\section{Conflicts of Interest}

The authors declare that there are no conflicts of interest.

\section{Acknowledgments}

Priscilla Naiff was supported by Amazonas State Research Support Foundation (FAPEAM). The authors acknowledge the National Council of Scientific and Technological Development (CNPQ), the Federal District Research Support Foundation (FAPDF), and SABIN laboratory for the financial support related to the research project developed by the postgraduation program in Health Sciences of the University of Brasilia (UnB), according to the Ph.D. thesis of Priscilla Farias Naiff, the main author of this article.

\section{References}

[1] World Health Organization, Global Report on Diabetes, World Health Organization, Geneva, Switzerland, 2016.

[2] A. M. Karande, R. Khandeparkar, and C. S. Vergeese, "Interrelationship between diabetes mellitus and periodontal disease based on their molecular mechanisms," Journal of Advanced Medical and Dental Sciences Research, vol. 5, pp. 24-32, 2017.

[3] A. Milech, A. P. Angelucci, A. Golbert et al., Diretrizes da Sociedade Brasileira de Diabetes 2015-2016, Sociedade Brasileira de Diabetes, AC Farmacêutica, São Paulo, Brazil, 2016.

[4] C. Dai, M. Brissova, Y. Hang, C. Thompson, G. Poffenberger, and A. Shostak, "Islet-enriched gene expression and glucoseinduced insulin secretion in human and mouse islets," Diabetologia, vol. 55, no. 3, pp. 707-718, 2012.

[5] A. Jindal, N. Agarwal, D. Sakalle, and P. Dushyant, "Diabetes mellitus and periodontitis-a two way relationship," PJSR, vol. 10, pp. 91-94, 2017.

[6] P. J. Ford, J. Gamonal, and G. Seymour, "Immunological differences and similarities between chronic periodontitis and aggressive periodontitis," Periodontology 2000, vol. 53, pp. 111-123, 2010.

[7] P. F. Naiff, P. P. Orlandi, and M. C. Dos-Santos, "Imunologia da periodontite crônica," Scientia Amazonia, vol. 1, pp. 28-36, 2012.

[8] O. Andrukhov, C. Ulm, H. Reischl, P. Nguyen, M. Matejka, and X. Rausch-Fan, "Serum cytokine levels in periodontitis patients in relation to the bacterial load," Journal of Periodontology, vol. 82, no. 6, pp. 885-892, 2011.
[9] J. Lindhe, T. Karring, and N. P. Lang, Tratado de Periodontia Clínica e Implantologia Oral, Guanabara Koogan, Rio de Janeiro, Brazil, 6th edition, 2018.

[10] G. U. Mohangi, S. Singh-Rambirich, and A. Volchansky, "Periodontal disease: mechanisms of infection and inflammation and possible impact on miscellaneous systemic diseases and conditions," SADJ, vol. 68, pp. 464-467, 2013.

[11] M. Soory, "Chronic periodontitis as a risk marker for systemic diseases with reference to cardiometabolic disorders: common pathways in their progression," Immunology and Immunogenetics Insights, vol. 2, pp. 7-21, 2010.

[12] S. Guzman, M. Karima, H. Y. Wang, and T. E. van Dyke, "Association between interleukin-1 genotype and periodontal disease in a diabetic population," Journal of Periodontology, vol. 74, no. 8, pp. 1183-1190, 2003.

[13] C. Tsai, C. Hayes, and G. W. Taylor, "Glycemic control of type 2 diabetes and severe periodontal disease in the US adult population," Community Dentistry and Oral Epidemiology, vol. 30, no. 3, pp. 182-192, 2002.

[14] A. D. Deshpande, M. Harris-Hayes, and M. Schootman, "Epidemiology of diabetes and diabetes-related complications," Physical Therapy, vol. 88, no. 11, pp. 1254-1264, 2008.

[15] J. B. Meigs, "Epidemiology of type 2 diabetes and cardiovascular disease: translation from population to prevention: the Kelly West award lecture 2009," Diabetes Care, vol. 33, no. 8, pp. 1865-1871, 2010.

[16] V. Lundberg, B. Stegmayr, K. Asplund, M. Eliasson, and F. Huhtasaari, "Diabetes as a risk factor for myocardial infarction: population and gender perspectives," Journal of Internal Medicine, vol. 241, no. 6, pp. 485-492, 1997.

[17] S. M. Haffner, S. Lehto, T. Ronnemaa, K. Pyorala, and M. Laakso, "Mortality from coronary heart disease in subjects with type 2 diabetes and in non-diabetic subjects with and without prior myocardial infarction," New England Journal of Medicine, vol. 339, no. 4, pp. 229-234, 1998.

[18] M. Laakso and S. Lehto, "Epidemiology of macro vascular disease in diabetes," Diabetes Reviews, vol. 5, pp. 294-315, 1997.

[19] F. B. Hu, M. J. Stampfer, C. G. Solomon et al., "The impact of diabetes mellitus on mortality from all causes and coronary heart disease in women: 20 years of follow-up," Archives of Internal Medicine, vol. 161, no. 14, pp. 1717-1723, 2001.

[20] M. Brownlee, "Advanced protein glycosylation in diabetes and aging," Annual Review of Medicine, vol. 46, no. 1, pp. 223-234, 1993.

[21] M. Sanz, A. Ceriello, M. Buysschaert et al., "Scientific evidence on the links between periodontal diseases and diabetes: consensus report and guidelines of the joint workshop on periodontal diseases and diabetes by the International Diabetes Federation and the European Federation of Periodontology," Diabetes Research and Clinical Practice, vol. 137, pp. 231-241, 2018.

[22] P. Chang and L. P. Lim, "Interrelationships of periodontitis and diabetes: a review of the current literature," Journal of Dental Sciences, vol. 7, no. 3, pp. 272-282, 2012.

[23] M. S. H. Akash, K. Rehman, and A. Liaqat, "Tumor necrosis factor-alpha: role in development of insulin resistance and pathogenesis of type 2 diabetes mellitus," Journal of Cellular Biochemistry, vol. 119, no. 1, pp. 105-110, 2018.

[24] P. P. Hujoel, B. A. White, R. I. Garcia, and M. A. Listgarten, "The dentogingival epithelial surface area revisited," Journal of Periodontal Research, vol. 36, no. 1, pp. 48-55, 2001.

[25] B. G. Loos, J. Craandijk, F. J. Hoek, P. M. Wertheim-van Dillen, and U. van der Velden, "Elevation of systemic markers 
related to cardiovascular diseases in the peripheral blood of periodontitis patients," Journal of Periodontology, vol. 71, no. 10, pp. 1528-1534, 2000.

[26] B. G. Loss, "Systemic markers of inflammation in periodontitis," Journal of Periodontology, vol. 76, no. 11-s, pp. 2106-2115, 2005.

[27] I. G. Ursãrescu, M. A. Martu-Stefanache, S. M. Solomon et al., "The assessment of Il-6 and RANKL in the association between chronic periodontitis and osteoporosis," Revista De Chimie, vol. 67, pp. 386-389, 2016.

[28] P. M. Ridker, "Clinical application of C-reactive protein for cardiovascular disease detection and prevention," Circulation, vol. 107, no. 3, pp. 363-369, 2003.

[29] S. K. Sonnenschein and J. Meyle, "Local inflammatory reactions in patients with diabetes and periodontitis," Periodontology 2000, vol. 69, no. 1, pp. 221-254, 2015.

[30] W. T. Y. Loo, Y. Yue, C. B. Fan et al., "Comparing serum levels of cardiac biomarkers in cancer patients receiving chemotherapy and subjects with chronic periodontitis," Journal of Translational Medicine, vol. 10, no. 1, pp. 1-7, 2012.

[31] G. J. Linden, A. Lyons, and F. A. Scannapieco, "Periodontal systemic associations: review of the evidence," Journal of Clinical Periodontology, vol. 40, pp. S8-S19, 2013.

[32] B. Chiu, "Multiple infections in carotid atherosclerotic plaques," American Heart Journal, vol. 138, no. 5, pp. S534-S536, 1999.

[33] J. Haraszthy, M. Zambon, M. Trevisan, and R. Zeid, "Identification of periodontal pathogens in atheromatous plaques," Journal of Periodontology, vol. 71, no. 10, pp. 1554-1560, 2000.

[34] E. Lalla, I. Lamster, M. Hofmann et al., "Oral infection with a periodontal pathogen accelerates early atherosclerosis in apolipoprotein E-null mice," Arteriosclerosis, Thrombosis, and Vascular Biology, vol. 23, no. 8, pp. 1405-1411, 2003.

[35] K. Nakano, H. Inaba, R. Nomura et al., "Detection of cariogenic Streptococcus mutans in extirpated heart valve and atheromatous plaque specimens," Journal of Clinical Microbiology, vol. 44, no. 9, pp. 3313-3317, 2006.

[36] L. K. Jennings, "Mechanisms of platelet activation: need for new strategies to protect against platelet-mediated atherothrombosis," Thrombosis and Haemostasis, vol. 102, no. 8, pp. 248-257, 2009.

[37] P. Ridker, C. Hennekens, J. Buring, and N. Rifai, "C-reactive protein and other markers of inflammation in the prediction of cardiovascular disease in women," New England Journal of Medicine, vol. 342, no. 12, pp. 836-843, 2000.

[38] P. Ridker and J. Silvertown, "Inflammation, C-reactive protein, and atherothrombosis," Journal of Periodontology, vol. 79, no. 8s, pp. 1544-1551, 2008.

[39] J. Beck and S. Offenbacher, "Oral health and systemic disease: periodontitis and cardiovascular disease," Journal of Dental Education, vol. 62, no. 10, pp. 859-870, 1998.

[40] M. Kebschull, R. T. Demmer, and P. N. Papapanou, “"Gum bug, leave my heart alone!"-epidemiologic and mechanistic evidence linking periodontal infections and atherosclerosis," Journal of Dental Research, vol. 89, no. 9, pp. 879-902, 2010.

[41] F. D'Aiuto, D. Ready, and M. S. Tonetti, "Periodontal disease and C-reactive protein-associated cardiovascular risk," Journal of Periodontal Research, vol. 39, no. 4, pp. 236-241, 2004.

[42] F. D'Aiuto, M. Parkar, G. Andreou et al., "Periodontitis and systemic inflammation: control of the local infection is associated with a reduction in serum inflammatory markers," Journal of Dental Research, vol. 83, no. 2, pp. 156-160, 2004.
[43] A. C. Leite, V. M. Carneiro, and M. C. Guimarães, "Effects of periodontal therapy on C-reactive protein and HDL in serum of subjects with periodontitis," Revista Brasileira de Cirurgia Cardiovascular, vol. 29, no. 1, pp. 69-77, 2014.

[44] B. L. Mealey and L. F. Rose, "Diabetes mellitus and inflammatory periodontal diseases," Current Opinion in Endocrinology, Diabetes and Obesity, vol. 15, no. 2, pp. 135-141, 2008.

[45] C. H. Peng, Y. S. Yang, K. C. Chan, E. Kornelius, J. Y. Chiou, and C. N. Huang, "Periodontal treatment and the risks of cardiovascular disease in patients with type 2 diabetes: a retrospective cohort study," Internal Medicine, vol. 56, no. 9, pp. 1015-1021, 2017.

[46] AAP, The American Academy of Periodontology, Proceedings of the World Workshop in Clinical Periodontics, AAP, Chicago, IL, USA, 1989.

[47] M. S. Tonetti and T. E. Van Dyke, "Periodontitis and atherosclerotic cardiovascular disease: consensus report of the joint EFP/AAP workshop on periodontitis and systemic diseases," Journal of Periodontology, vol. 84, no. 4-s, pp. S24-S29, 2013.

[48] Y. W. Fu, X. X. Li, H. Z. Xu, Y. Q. Gong, and Y. Yang, "Effects of periodontal therapy on serum lipid profile and proinflammatory cytokines in patients with hyperlipidemia: a randomized controlled trial," Clin Oral Investig, vol. 20, no. 6, pp. 1263-1269, 2016.

[49] J. Ainamo and I. Bay, "Problems and proposals for recording gingivitis and plaque," International Dental Journal, vol. 25, pp. 229-235, 1975.

[50] F. D’Aiuto, M. Parkar, G. Andreou, P. M. Brett, D. Ready, and M. S. Tonetti, "Periodontitis and atherogenesis: causal association or simple coincidence?," Journal of Clinical Periodontology, vol. 31, no. 5, pp. 402-411, 2004.

[51] P. M. Duarte, M. da Rocha, E. Sampaio et al., "Serum levels of cytokines in subjects with generalized chronic and aggressive periodontitis before and after non-surgical periodontal therapy: a pilot study," Journal of Periodontology, vol. 81, no. 7, pp. 1056-1063, 2010.

[52] T. Nakajima, T. Honda, H. Domon et al., "Periodontitisassociated up-regulation of systemic inflammatory mediator level may increase the risk of coronary heart disease," Journal of Periodontal Research, vol. 45, no. 1, pp. 116-122, 2010.

[53] H. P. C. Artese, A. M. Foz, M. D. S. Rabelo et al., "Periodontal therapy and systemic inflammation in type 2 diabetes mellitus: a meta-analysis," PLoS One, vol. 10, no. 5, Article ID e0128344, 2015.

[54] S. Gopalakrishnan, T. Ramakrishnan, P. Harinath, J. Moses, V. Shankarram, and S. Raj, "Effects of non-surgical periodontal therapy on plasma level of reactive oxygen metabolites and glycemic status in type-2 diabetic patients with chronic periodontitis," Biosciences, Biotechnology Research Asia, vol. 14, no. 1, pp. 357-365, 2017.

[55] A. F. Stadler, P. D. Angst, R. M. Arce, S. C. Gomes, R. V. Oppermann, and C. Susin, "Gingival crevicular fluid levels of cytokines/chemokines in chronic periodontitis: the meta-analysis," Journal of Clinical Periodontology, vol. 43, no. 9, pp. 727-745, 2016.

[56] M. Claudino, G. Gennaro, T. M. Cestari, C. T. Spadella, G. P. Garlet, and G. F. Assis, "Spontaneous periodontitis development in diabetic rats involves an unrestricted expression of inflammatory cytokines and tissue destructive factors in the absence of major changes in commensal oral microbiota," Experimental Diabetes Research, vol. 2012, p. 10, 2012. 
[57] P. N. Madianos and P. A. Koromantzos, "An update of the evidence on the potential impact of periodontal therapy on diabetes outcomes," Journal of Clinical Periodontology, vol. 45, no. 2, pp. 188-195, 2018.

[58] D. Polak and L. Shapira, "An update of the evidence for pathogenic mechanisms that may link periodontitis and diabetes," Journal of Clinical Periodontology, vol. 45, no. 2, pp. 150-166, 2018.

[59] B. M. Mishriky, D. M. cummings, and R. J. Tanenberg, "The efficacy and safety of DPP4 inhibitors compared to sulfonylureas as add-on therapy to metformin in patients with Type 2 diabetes: a systematic review and meta-analysis," Diabetes Research and Clinical Practice, vol. 109, no. 2, pp. 378-388, 2015.

[60] P. Sharma, T. Dietrich, C. J. Ferro, P. Cockwell, and I. L. C. Chapple, "Association between periodontitis and mortality in stages 3-5 chronic kidney disease: NHANES III and linked mortality study," Journal of Clinical Periodontology, vol. 43, no. 2, pp. 104-113, 2016. 


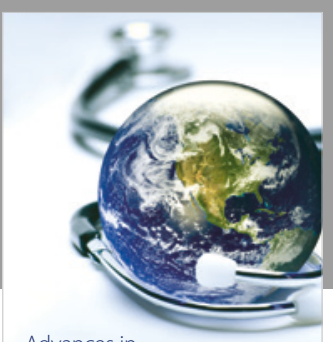

Advances in
Public Health

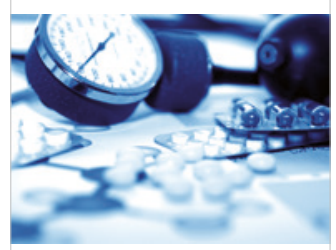

Case Reports in

Medicine

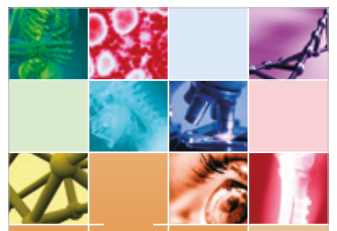

niernational Journal of

Biomaterials
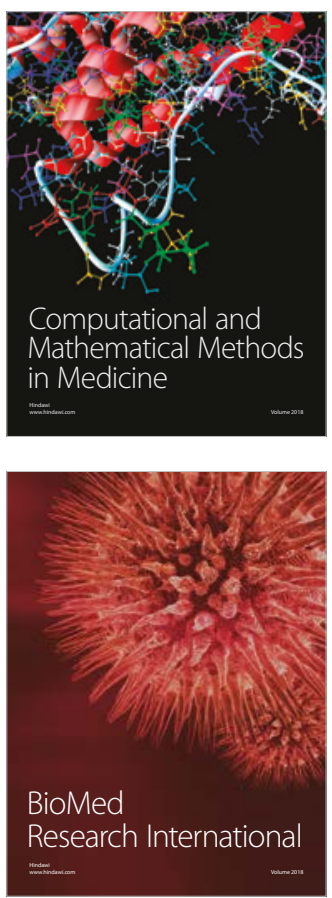

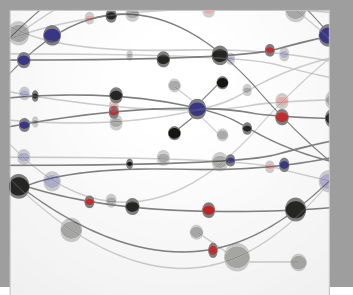

The Scientific World Journal Dentistry

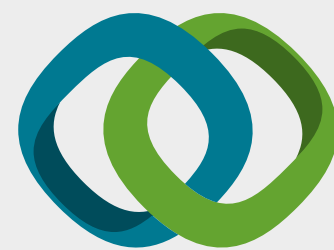

Hindawi

Submit your manuscripts at

www.hindawi.com
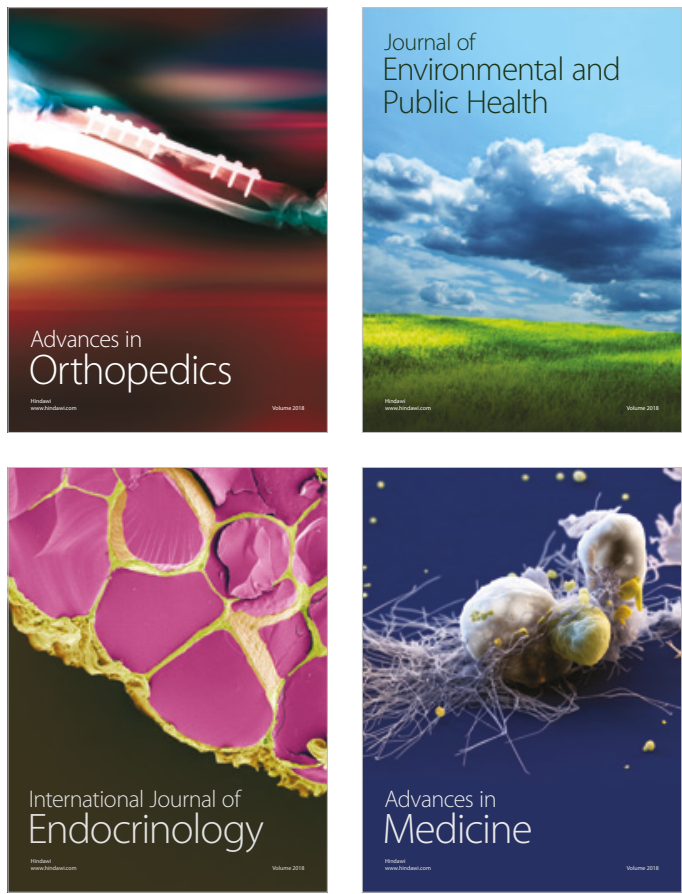
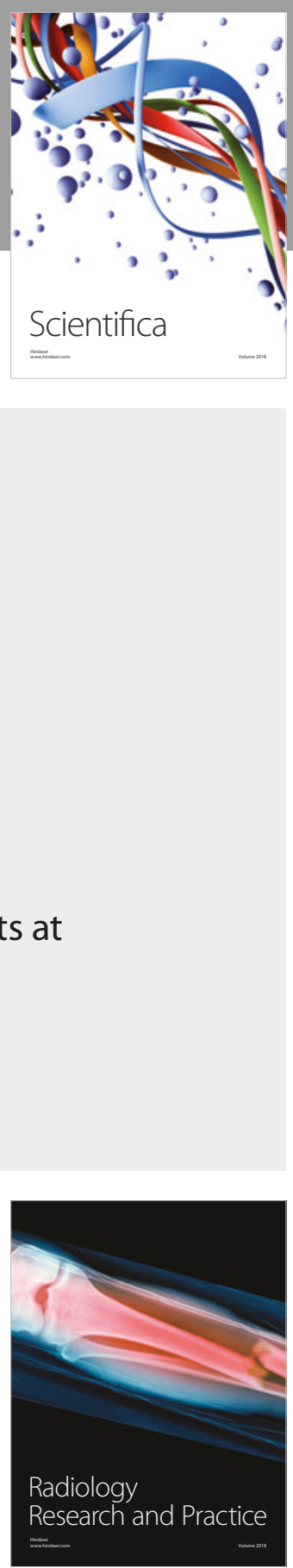

Scientifica

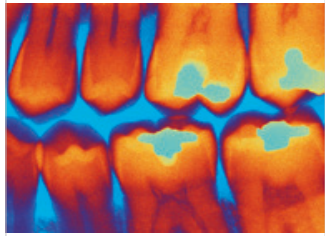

Case Reports in

Dentistry
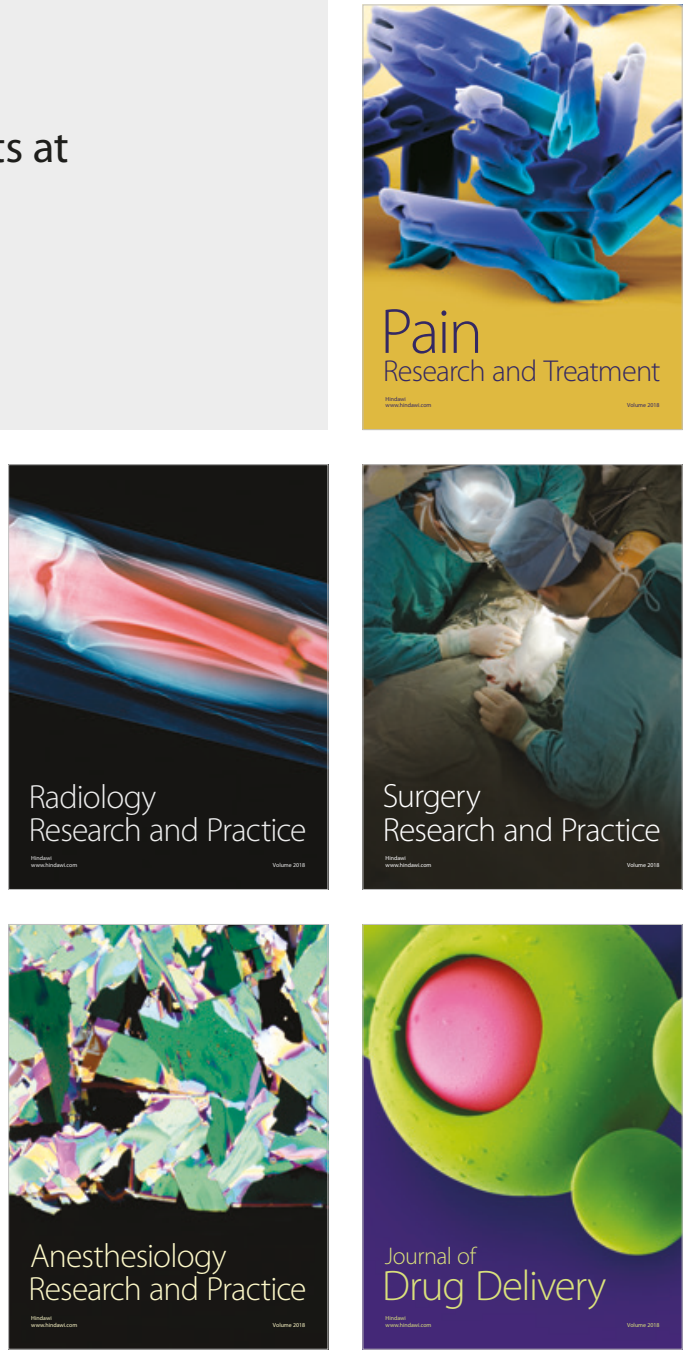